\title{
Polymer Fiber Optic Sensors - A Mini Review of their Synthesis and Applications
}

\author{
Yan Jin and Anthony M Granville*
}

Centre for Advanced Macromolecular Design, School of Chemical Engineering, University of New South Wales, Sydney, Australia

\begin{abstract}
Fiber optic sensors (FOSs) are optical fiber based sensing devices that have been widely used to detect physical, chemical and biological parameters. Polymer optical fibers (POFs), an important type of optical fibers, have been stifled their talents in the field of optical sensing due to the overwhelming popularity of glass optical fibers (GOFs). The unique physical and chemical properties of POFs start attracting interests to be utilized in the fabrication of high performance sensors in recent years. This article reviews the recent progress of POFs based FOSs. In particular, the article will focus on novel techniques implemented on the POF sensor fabrication, including monolayer graphene, evanescent wave absorption, localized surface plasmon resonance and fiber Bragg grating.
\end{abstract}

Keywords: Polymer fiber optic sensor; Evanescent wave; Localized surface plasmon resonance; Fiber Bragg gratings; Microstructured polymer optical fiber

\section{Introduction}

Although it is an essential part of modern communication, the light guiding capabilities of optical fibers were first demonstrated in the early $1840 \mathrm{~s}$ by Daniel Colladon and Jacques Babinet who showed that light can be guided through the glass fiber by the total internal reflection (TIR) at the fiber's inner surface [1]. Fiber optic sensors (FOSs) operate on the principle that the light transmitted through the fiber core can be affected by its surrounding environment [2]. Over the years, a range of FOSs have been developed to measure a variety of parameters, including physical (displacement, stress and wind speed) [3-5], chemical ( $\mathrm{pH}$, organic and inorganic compound concentrations) and biological parameters (blood glucose and oxygen concentrations) $[6,7]$. To date, FOSs are frequently developed using glass optical fibers (GOFs) due to their low attenuation of light signals and commercial availability [8]. However, the limitations for developing sensors from GOFs have usually, but not exclusively, been due to the demanding procedures for fiber processing rather than their performance [9]. On the other hand, polymer optical fibers (POFs), with structure and light guiding properties comparable to GOFs, are receiving increased attention from researchers due to their high flexibility and ease of handling $[2,6,10]$. In particular, the surface properties of POFs can be easily manipulated by simple and wide-ranging chemistry techniques to achieve the desired sensing properties.

The relevant theories of fiber optical sensors have been thoroughly explained by Udd [11] and López-Higuera [12]. Early reviews of polymer optical fibers sensors are included in some general reviews of fiber optic sensors, including Grattan [13], Zubia [2], Bartlett [14] and Leung [15]. Recently, more specific reviews on polymer fiber based optic sensors are given by Peters [16] and Bilro [8]. This paper presents a brief review of the recent developments in POF sensors and their applications, specifically, the most common fabrication techniques and sensing mechanisms will be demonstrated and discussed.

\section{Polymer optical fibers}

POFs were developed nearly at the same time as the GOFs, but they have received far less attention compared to GOFs. The relatively high signal attenuation of POFs makes them unsuitable for long-haul communications. However, their ease of handling, greater flexibility and high robustness make POFs ideal for short-haul communications and sensing applications [2]. Since the invention of POFs, continuous efforts have been devoted to POFs technologies and developments to improve its light guiding properties. Several types of POFs have been developed thus far, including single/multi-mode POF (SM/MM-POF), step-index POF (SI-POF), graded-index POF (GI-POF), fluorinated POF (CYTOP) and the latest multi-core POF $[2,17]$.

The structure of a common MM-SI POF consists of three layers: core, cladding and jacket (Figure 1). The refractive index of the cladding $\left(n_{c l}\right)$ is designed to be lower than that of the core $\left(n_{c o}\right)$, so it can facilitate TIR in the fiber core. Several core materials have been utilized for POFs such as poly (methyl methacrylate) (PMMA), polystyrene (PS) and polycarbonate (PC). Each of the core materials has its own unique characteristics and applications. For example, PMMA POFs have relatively high transparency in visible and near-infrared regions and hence they are the most common POFs on the market, while PC POFs have better heat resistance compared to PMMA POFs and hence they can be used to fabricate sensors in a high temperature environment [2]. The jacket is a plastic layer on the cladding which provides protection for the fiber from chemical decomposition and mechanical damages. It is worth noting that, in some cases such as POFs for sensing applications, uncladded POFs (without the cladding and the jacket) are frequently used as the sensing events occur on the surface of the fiber core.

Over the years, technological advances have allowed the production of high-quality POFs at lower cost. Currently, two main techniques have been widely adopted for producing SM/MM-SI POFs: drawing process and extrusion process [18].

*Corresponding author: Anthony $M$ Granville, Centre for Advanced Macromolecular Design, School of Chemical Engineering, University of New South Wales, Sydney, Australia, Tel: +61 29385 5745; Fax: +61 29385 5966; E-mail: a.granville@unsw.edu.au

Received November 17, 2015; Accepted December 10, 2015; Published January 01,2016

Citation: Jin Y, Granville AM (2016) Polymer Fiber Optic Sensors - A Mini Review of their Synthesis and Applications. J Biosens Bioelectron 7: 194. doi:10.4172/21556210.1000194

Copyright: (c) 2016 Jin Y, et al. This is an open-access article distributed under the terms of the Creative Commons Attribution License, which permits unrestricted use, distribution, and reproduction in any medium, provided the original author and source are credited. 


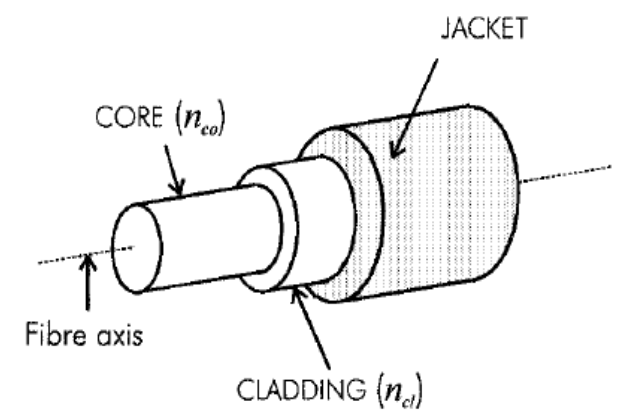

Figure 1: The structure of a modern optical fiber [2]. Reprinted with permission from Elsevier, copyright 2001.

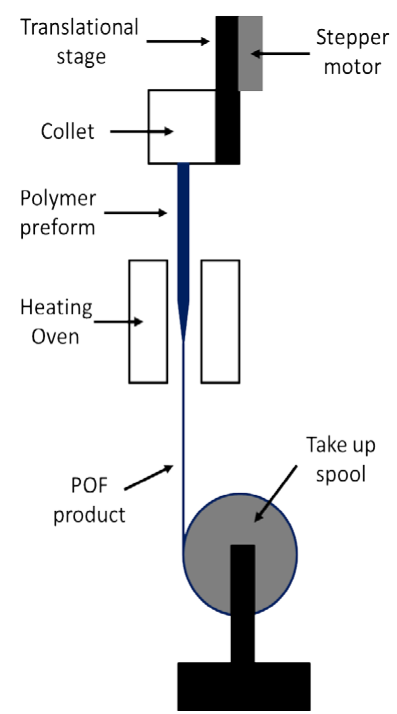

Figure 2: Schematic diagram of a POF drawing tower.

\section{Drawing process}

Garvey et al. described the general procedures for the POF drawing process [19]. First, a cylindrical shape of polymer preform is obtained with a typical diameter of $0.5-1 \mathrm{~m}$. The preform obtained has the same index profile as the fiber core being drawn. Next, the preform is transferred to a fiber drawing tower, where it is drawn into a smaller fiber with a predetermined diameter. Figure 2 illustrates a typical drawing tower: a stepper motor holds the preform vertically and feeds it into an oven. The optimal heating condition varies with the preform material and is usually determined empirically. In the oven, the polymer becomes soft and forms a neck-down region. The fiber below the neck-down region can be extruded into a smaller fiber, while the diameter of a POF is controlled by a three-pulley stress gauge system (not shown in the figure) [18]. The fiber exits the tension gauge system by a take-up spool and ready for further processing. The drawing process is a rather simple operation and can be used for production of various kinds of POFs using the corresponding polymer performs that are commercially available. The main problem associated with this process arises from variations of drawing conditions during the manufacturing process that could change the index of fiber from one section to another [19].

\section{Extrusion process}

Compared to the drawing process, the extrusion process produces POFs directly from monomers either continuously or in batches. The extrusion process can be performed under air or in a closed environment (thrust extrusion) [17]. Compared to the open air extrusion process, thrust extrusion prevents any impurities which influence the optical properties of the final POFs.

Figure 3 shows a schematic diagram of a typical thrust extrusion process for producing PMMA POFs [20]. At the beginning of the process, monomer, initiators and other necessary substances are distilled into a closed reactor under vacuum to minimize the amount of air dissolved in the system. The polymerization is carried out at $135^{\circ} \mathrm{C}$ for $12 \mathrm{~h}$ and then raised to $180^{\circ} \mathrm{C}$ for another $12 \mathrm{~h}$ to achieve a high conversion of the monomer [18]. After the polymerization process is completed, nitrogen is used to generate high pressure in the reactor to push the melted polymer through a small nozzle, producing the desired fibers. The remaining monomers can be evaporated and recovered back into the feedstock. Although the thrust extrusion process produces higher quality POFs than that produced from the drawing process, the higher requirements for equipment and operations limit its applications to mainly industrial scale POF productions.

\section{Polymeric optical fiber sensors}

Fiber optic sensors can be classified into several categories depends on their configurations, working mechanisms and applications. One of the generally accepted classifications is extrinsic and intrinsic sensors (Figure 4). In an extrinsic sensor, the optical signal leaves the waveguide

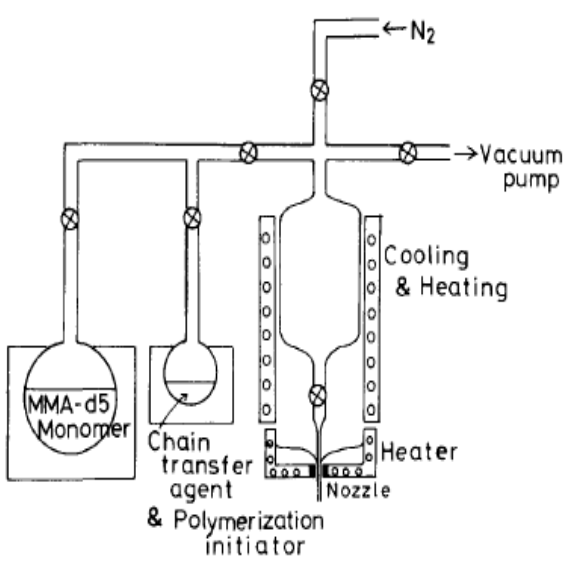

Figure 3: Schematic diagram of a PMMA POF extrusion apparatus [20]. Reprinted with permission from AIP Publishing LLC, copyright 1982.

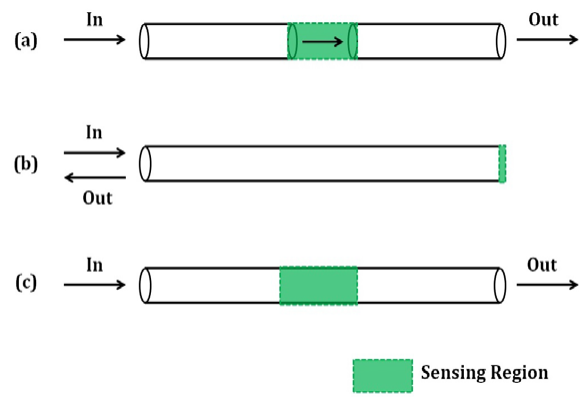

Figure 4: (a) Extrinsic system, (b) intrinsic-reflection system, (c) intrinsictransmission system. 
first, entering a sensing region and then re-enters the second waveguide. On the other hand, for an intrinsic sensor, the optical signal does not leave the waveguide, it is either reflected back to the entering side (reflection system) or transmitted to the other side of the waveguide (transmission system). For intrinsic sensors, a reflection system utilizes absorption, fluorescence, light scattering, refractive index and intensity variations etc. to detect target parameters. On the other hand, transmission systems can further take advantage of evanescent wave absorption to monitor changes occurring on the surface of the fiber. Since transmission systems have the capability to fully explore the large side-surface area of the optical fibers, thus the following review will focus on intrinsic sensors.

\section{Intrinsic-reflection POF sensors}

Intrinsic-reflection systems are the conventional configuration for the fabrication of fiber optic sensors. Among the intrinsic-reflection systems reported to date, the majority employ indicators at the fiber ends which respond to the parameters to be detected. Indicators employed in intrinsic-reflection POF sensors are frequently doped in a thin film and subsequently dip-coated (e.g. sol-gel technique) or physically attached onto the fiber end. Thin film techniques have been well investigated and developed for the fabrication of GOF sensors during the last few decades and these technologies have been successfully transferred to fabricate POF sensors.

Utilizing sol-gel technology to create a thin porous film incorporating sensing elements for optical sensors, especially biosensors, has attracted considerable interest. Thin films on the fiber ends with a desired layer thickness can be easily fabricated through a simple process which includes spin-coating, dip-coating and spraycoating [21]. By carefully tailing the supporting materials, fabrication process and layer configuration, the thin film can achieve a high compatibility with biological molecules, such as enzymes, DNA and even whole cells. The relevant theory of sol-gel techniques has been thoroughly discussed by Jerónimo and MacCraith [22].

Fiber optic sensor for $\mathrm{pH}$ sensing and imaging is the field where sogel techniques have been most frequently implemented. This is due to the convenience of incorporating $\mathrm{pH}$ indicators into the sol-gel matrix, which, compared to conventional $\mathrm{pH}$ sensors, offers several advantages such as fast response, high resolution, low cost and immunization of EMI. Fabbri and Rovati reported a disposable $\mathrm{pH}$ sensor based on an organic-inorganic hybrid matrix-ORMOSILs (organically modified silicates) that were doped with a $\mathrm{pH}$ sensitive dye [23]. Polyethylene oxide (PO) was used as the organic fraction of the matrix, which allows for good adhesion between the POF and the porous matrix. It also can be used to tune the diffusion rate of the analyte inside the porous matrix. The inorganic fraction of the matrix-silica glass (prepared from tetraethyl orthosilane, TEOS)-enhances the ductility and permeability of the matrix. The hybrid matrix was obtained from a typical sol-gel technique and then dip-coated on the tip of a PMMA fiber. The sensing range was found to be $\mathrm{pH} 4-10$ with less than 1 second response time. Later on, Schryy et al. adopted a similar strategy using TEOS and methyltriethoxysilane (MTES) to prepare an organic-inorganic matrix that supports bromophenol blue (BB) to monitor $\mathrm{pH}$ in biological fluids [24]. The optimum amount of organic compound-MTES was found to be $50 \mathrm{wt} \%$ which reduced dye leaching in phosphate buffered saline (PBS) within $20 \%$ for the duration of 2 months. The fabricated sensor showed good sensitivity in the range of $\mathrm{pH} 5-8$, and temperature independence within 20 to $25^{\circ} \mathrm{C}$. Furthermore, in vitro tests in human serum showed reversible $\mathrm{pH}$ monitoring between $\mathrm{pH}$ 5-8 with $0.2 \mathrm{pH}$ unit resolutions.
Along with $\mathrm{pH}$ sensing, chemical aerosol detection has been performed by Kulkarni et al. testing the possibility of using two types of thin film configurations, TEOS and TEOS doped with thylmol blue (TB) [25]. By utilizing a typical sol-gel technique, a thin layer of TEOS was doped onto the end surface of a POF. The fabricated POF sensor was equipped with 4 types of light sources: $U V(\lambda=340 \mathrm{~nm})$, blue $(\lambda$ $=440 \mathrm{~nm})$, red $(\lambda=660 \mathrm{~nm})$ and infrared $(\lambda=940 \mathrm{~nm})$, which were used to detect black carbon (BC) and sulfate- $\left(\mathrm{NH}_{4}\right)_{2} \mathrm{SO}_{4}$ aerosols. The preliminary testing results showed that $\mathrm{TB}$ doped films demonstrated a better sensitivity than pure TEOS films and blue light sources led to a more significant intensity change due to the stronger absorption by aerosols: $2.22 \%$ for sulfate and $2.16 \%$ for BC. The authors also pointed out that, by carefully controlling the thickness of thin film, the sensitivity could be further improved.

Portable, low cost and fast detection of nitro aromatic explosives, such as trinitrotoluene (TNT) and trinitrophenol (TNP) remain a critical importance for safety reasons. The discovery of fluorescein conjugated-poly(2-methoxy-(2'-ethylhexloxy)-p-phenylene-vinylene) (MEH-PPV) enables fast identification of these explosives due to its fluorescence quenching effect when in contact with nitro aromatic compounds [26]. Chu et al. reported a TNT POF sensor by dip coating MEH-PPV on a coil-shaped PMMA fiber [27]. It was discovered that better sensitivity could be achieved by measuring the fluorescent life time rather than the intensity, while the sensitivity was also improved for the fibers with a smaller coil. However, the sensor's lowest detection limit (1-15 ng. $\left.\mathrm{mL}^{-1}\right)$ was extrapolated from the sensor's response curve, which needs to be verified by experimental tests. Irreversible quenching of using MEH-PPV by TNT is one of the main drawbacks of the sensor, meaning that it is not suitable for in vitro measurements.

Graphene, the two-dimensional nanomaterial, is a good candidate to be used as the sensing elements in the application of fiber optic sensing technologies. The properties and production technique of graphene have been reported in some comprehensive reviews [28-30]. The characteristics of ultra-low thickness, high permeability, good optical transparency as well as chemical inertness make graphene films ideal carriers for binding of transducer reagents in integrated biochemical transducer fiber optic systems [31-34]. Currently, the application of graphene in FOSs has been mostly focused on GOFs, while a limited number of POF based FOSs has been reported [35-37].

Kulkarni et al. first reported a simple strategy to transfer large area graphene films onto the distal end surface of PMMA fibers by direct deposition of preformed graphene films synthesized via a chemical vapor deposition (CVD) technique [36]. This technique was later implemented by the same research group who designed a volatile organic compounds (VOCs) sensor via depositing a monolayer of graphene or graphene oxide onto the distal end of a PMMA fiber [37,38] The intensity of light reflected from the deposited thin film will change accordingly when the film absorbs VOCs present in the atmosphere. Recently, POFs with their claddings replaced with graphene films have demonstrated the capability to detect biomolecules in aqueous mediums by monitoring the shift of surface plasmon resonance (SPR) [35].

\section{Intrinsic-transmission POF sensors}

The highly restricted sensing area (fiber distal-end) of intrinsicreflection systems significantly hinders the efforts to improve their sensing performance. Meanwhile, the emergence of new types of POFs (e.g. microstructured POFs and fiber Bragg gratings) and detection mechanisms (e.g. evanescent wave absorption and localized surface 
plasmon resonance) provide intrinsic-transmission systems with much greater potentials due to their detection diversity and sensitivity. For that reason, intrinsic-transmission systems have dominated the research of FOSs in recent years.

Evanescent wave absorption (EWA) POF sensors have been receiving increasing attention from researchers due to their potential medical applications in detecting biomolecules [39-41]. When light is transmitted through the fiber, it undergoes total internal reflection on the inner surface of the fiber core. This internal reflection gives rise to the standing wave at the interface along the axis of the fiber due to the interference between incident and reflected beams. Since the amplitude of the standing wave does not decay to zero at the boundary between the fiber core and the surrounding medium, it penetrates a short distance into the surroundings. This "leakage" of light from the wave guide is defined as an evanescent wave (EW). The intensity of the EW present on the fiber surface can be expressed in the following equation [42]:

$$
\mathrm{E}=\mathrm{E}_{0} \mathrm{e}^{-\delta / \mathrm{d}_{\mathrm{p}}}
$$

Where, $\mathrm{E}_{0}$ is the power of light coupled into the fiber, $\mathrm{E}$ is the power of the EW, $\delta$ depicts the distance from the interface and $d_{p}$ stands for the penetration depth of the $\mathrm{EW}$ where the amplitude of the intensity fall to $1 / \mathrm{e}$ its value at the interface [42].

$$
\mathrm{d}_{\mathrm{p}}=\frac{\lambda}{2 \pi\left(\mathrm{n}_{\mathrm{co}}^{2} \sin ^{2} \Theta-\mathrm{n}_{\mathrm{cl}}^{2}\right)^{\frac{1}{2}}}
$$

Penetration depth- $\mathrm{d}_{\mathrm{p}}$ depends on several parameters: the wavelength of coupled light $(\lambda)$, the refractive indexes of core $\left(n_{c o}\right)$, surrounding mediun $\left(\mathrm{n}_{\mathrm{cl}}\right)$, and incident angle $(\theta)$ between the ray and the normal interface.

The EW interacts with analytes in the vicinity of fiber surface via absorption of EW at certain wavelengths or emission of light after EWA (luminescence) (Figure 5) [17]. By analyzing the output light spectrum, the fingerprints of solids, liquids and gases that present on the surface can be obtained.

In most cases, the target analyte cannot be detected directly because its interaction with the EW does not always lead to a detectable spectra change (e.g. weak absorption of EW). The common solution is to use indicators that can interact with analytes, producing a certain detectable change in the micro-environment of the fiber surface [43]. One of the most commonly employed indicators is metal nanoparticles of noble metals. When excited by an EW, the nanoparticles immobilized on the fiber surface will be excited to trigger a well-known optical phenomenon called localized surface plasmon resonance (LSPR) which exhibits ultrahigh sensitivity towards the variation of refractive index in the vicinity of the particle surface $[44,45]$. LSPR effect and the corresponding LSPR

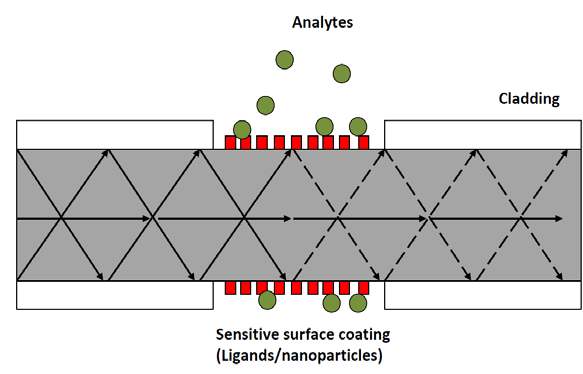

Figure 5: Operating principles of the surface modified POF sensors.
POF sensors lay the foundation for the research project reported in this thesis hence these topics will be further explored individually in subsequent sections.

One of the basic applications of tapered EWA POF sensors is to detect the salinity of salt water as the increase of medium refractive index would result in the reduction of the light intensity transmitted through the fiber [46]. However, the sensor response to bulk refractive index variations caused by salinity changes due to the unspecific detection mechanism. The EWA salinity POF sensor does not provide significant detection improvement over conventional electrical sensors [47].

More advanced development of EWA POF sensors involves the implementation of an indicator. Pulido et al. reported a EWA POF oxygen sensor based on the fluorescence-quenching effect of oxygen on cationic ruthenium complexes [48]. Tris (2,2'-bipyridyl) dichlororuthenium(II) hexahydrate was first dissolved in the solvent of acetone and water, followed by immersing the tapered section of PMMA POF in the solution for a short period of time. Due to the partial surface dissolution of PMMA POF caused by acetone, the ruthenium complex was able to diffuse into the upper layers of the PMMA POF and being trapped. The reported sensor showed linear responses to oxygen concentration from $0-100$ vol\% both in terms of fluorescence intensity and fluorescence lifetime. However, the immersion time of PMMA POFs in the acetone and water mixture has to be carefully controlled as the mechanical stability of PMMA POFs degraded rapidly in the solution. A sol-gel technique was also employed to anchor ruthenium complexes onto a U-shape polymer fiber for the fabrication of a dissolved oxygen sensor [49]. Ruthenium (II) chloride was incorporated into a sol-gel prepared from TEOS and MTEOS and then dip-coated onto a U-shape de-cladded PMMA fiber. However, non-linear responses were observed for both fluorescence intensities and lifetimes against oxygen concentrations. The authors proposed a two-layer model to explain this non-linearity: the sensing film consists of two layers, the outer layer contacts the dissolved oxygen directly while the inner layer does not. With the proposed two-layer model and the previously reported dynamic/static quencher model combined [50], the new model is more comprehensive in explaining the non-linear behavior of the experimental data.

A tapered EWA POF biosensor was report by da Silva et al. for the detection of red blood cells (RBC) and bacterium Escherichia coli [51]. A small region of a PMMA POF ( $1 \mathrm{~mm}$ in diameter) was heated above its glass transition temperature and pulled to form a tapered section (0.35 $\mathrm{mm}$ in diameter) with a biconic format. Then, hexamethylene diamine (HMDA) and glutaraldehyde were applied to the tapered region followed by protein $\mathrm{A}$ for the subsequent immobilization of anti-RBC and anti-E.coli. An LED light source $(540 \mathrm{~nm})$ was irradiated into the fiber which was further split into two channels: one entered the sensing fiber and the other one entered the reference fiber. The testing results were positive for $\mathrm{RBC}$ at concentrations of $10^{8}$ colony forming units. $\mathrm{mL}^{-1}\left(\mathrm{CFU} \cdot \mathrm{mL}^{-1}\right)$ while failed for anti-E.coli due to the weak absorbance at $540 \mathrm{~nm}$. However, E.coli was successfully detected at a minimum concentration of $10^{4} \mathrm{CFU} \cdot \mathrm{mL}^{-1}$ by switching the emission source to longer wavelengths $(880 \mathrm{~nm})$ due to the stronger EW intensity presented on the fiber surface [52]. Although the sensitivity of the reported EWA POF biosensor was considerably lower than the methods currently available on the market (e.g. polymerase chain reaction technology can achieve $<10^{2} \mathrm{CFU} \cdot \mathrm{mL}^{-1}$ ) [53], the continuous improvements of the EWA POF biosensor would certainly make it more competitive in the near future. 


\section{Intrinsic-transmission POF sensors using Fiber Bragg Gratings}

Fiber Bragg grating (FBG) sensors are a type of wavelengthencoded sensor. FBG-based sensing devices directly transform sensed parameters into shifts of optical wavelengths, which have the advantage of minimal influence caused by extraneous optical power variations. According to the theory of Fresnel reflection, the light wave can reflect and refract at the interface of the two mediums which have different refractive indices. By varying the refractive index of the fiber core periodically (using laser or UV light), FBGs can act as a wavelengthspecific dielectric mirror that reflect certain wavelengths of the input light and transmit through the rest of the wavelengths [54]. Figure 6 illustrates a uniform positive index change FBG which is the simplest form of FBG that is employed most frequently in the FBG sensor fabrication.

$$
\lambda_{\mathrm{B}}=2 \mathrm{n}_{\mathrm{e}} \Lambda
$$

The light guiding properties of a FBG is governed by Equation 3 [54], where $n_{e}$ is the effective refractive index of the grating in the fiber core, $\lambda_{B}$ is the Bragg wavelength and $\Lambda$ is the grating period. The sensing capabilities of FBG sensors are determined by $n_{e}$ and $\lambda_{B}$. When external stimuli are applied to a FBG sensor, such as strain, pressure, humidity and temperature variations, $\mathrm{n}_{\mathrm{e}}$ and $\Lambda$ would change accordingly and result in the shift of $\lambda_{B}$. Therefore, all FBG sensors are intrinsically sensitive to the aforementioned stimuli [55]. Recently, extension of the sensing capability of FBG sensors to detect biological agents has been reported by modifying the fiber grating surface which induces binding of biological analytes. Once analytes bind onto the fiber grating surface, they change the surface strain of the fiber and result in a shift of the Bragg wavelengths [56-58]. However, due to the interference caused by the surrounding environment, such as humidity and temperature variations, the performance of FBG biosensors is inferior compared to other types of fiber optic biosensors and a major breakthrough is still required. The following section will mainly focus on the well-developed FBG sensors to detect physical parameters.

Using POFs to fabricate FBG sensors offers several advantages over GOFs. The lower Young's modulus of POFs leads to a higher failure strain which results in an improved sensitivity and detection range. Additionally, by taking advantages of the high flexibility and easy processing of POFs, asymmetric structure of polymer based FBG sensors can be fabricated to achieve certain functionalities. The first polymer FBG was fabricated by Xiong et al. [59]. FBG was incorporated into a SM PMMA POF using a pulse laser beam operating at 248 $\mathrm{nm}$. After that, several polymer FBGs were developed with different Bragg wavelengths, dopants and fiber geometries [60-63]. Recently, more attention has been drawn to inscribing Bragg gratings into microstructured polymer optical fibers (mPOFs) due to their superior

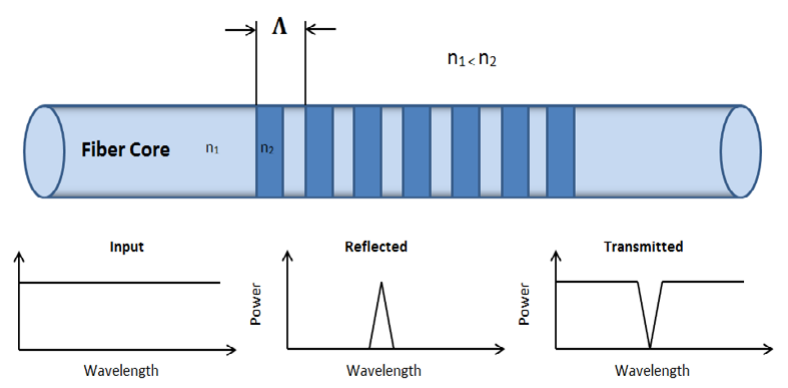

Figure 6: Uniform positive index change FBG. properties, which will be discussed in the following section [64-67].

Strain, temperature and humidity measurements are one of the most common fields in which polymer FBG sensors have so far been implemented. Luo et al. evaluated the performance of a polymer FBG when measuring stress and strain [68]. The authors inscribed gratings into a SI-MM POF with the core doped with benzyl dimethyl ketal (BDK) to enhance the photosensitivity. A $355 \mathrm{~nm}$ frequency-tripled Nd: YAG laser was used to inscribe gratings into POFs with Bragg wavelength of $1570 \mathrm{~nm}$. The maximum sensitivity of axial stress was determined to be $472 \mathrm{pm} \cdot \mathrm{MPa}^{-1}$ under $9.59 \mathrm{MPa}$ stress, while the sensitivity of axial strain was comparable to GOF based FBG sensors $\left(1.18 \mathrm{pm} \cdot \mu \varepsilon^{-1}\right)$. Later on, the same research group extended the use of the polymer FBG sensor in measuring temperature [69]. Both theoretical simulation and experimentation showed that the MM polymer FBG sensor can achieve a higher sensitivity by an order of magnitude than their glass FBG counterparts.

Besides the commonly adopted techniques that use laser or ion beams to inscribe Bragg gratings into POFs or GOFs, $\mathrm{Gu}$ et al. demonstrated a high strain sensitivity polymer micro/nanofiber Bragg gratings (MNFBGs) employing a low cost nanoimprinting approach [70]. Similar to the technique adopted in the fabrication of gratings in planar waveguides, they used a standard plane reflection grating as a mold to imprint gratings on a POF when it was heated above its glass transition temperature. The axial strain sensitivity of the fabricated FBG sensor was determined to be $-2.5 \mathrm{pm} \cdot \mu \varepsilon^{-1}$ which is almost doubled when compared to common polymer FBG strain sensors [68]. One apparent difference of this FBG sensor is the blue shift of the Bragg wavelength upon applying strain, in contrast to the response of FBG sensors fabricated using laser or ion beams. The authors believe this contradiction was resulted from a large photo-elastic effect when applying strain, while the red shifts from conventional FBG sensors were caused by the elongation of the fibers.

The realization of multiplex sensing in FBG sensors, especially in measuring physical parameters, is much easier than other FOSs. Bragg gratings were reported by writing into an eccentric core polymer optical fiber (ecPOF) to fabricate a strain, bend and temperature sensor [71]. Compared to concentric polymer fibers, ecPOF has characteristics of directional bend sensitivity and wide curvature measurement range. The POF used for sensor fabrication was a SI-SM PMMA fiber with a core offset of $24 \mu \mathrm{m}$ from the neutral axis, the diameters of the core and the cladding was $8.1 \mu \mathrm{m}$ and $230 \mu \mathrm{m}$, respectively. The Bragg grating was inscribed into ecPOF via a common scanning phase mask technique using a CW He-Cd laser operated at $325 \mathrm{~nm}$. The bend experiments were carried out for four orientations individually. For each rotational orientation, the Bragg peak shift was monitored for curvatures ranging from 0 to $22.7 \mathrm{~m}^{-1}$. The maximum sensitivities were found to be $63.3 \mathrm{pm} \cdot \mathrm{m}^{-1}$ at $180^{\circ}$ orientation (extension) and -56.4 $\mathrm{pm} \cdot \mathrm{m}^{-1}$ at $0^{\circ}$ orientation (compression). The strain and temperature tests showed the FBG in an ecPOF had a maximum sensitivity of 1.13 $\mathrm{pm} \cdot \mu \varepsilon^{-1}$ and $-50.1 \mathrm{pm} \cdot{ }^{\circ} \mathrm{C}^{-1}$.

Polymer FBG sensors can respond to the humidity variations in the surrounding environment when water from the atmosphere diffuses into the fiber. Zhang et al. compared the performance of POF and GOF based FBGs in measuring humidity and temperature [72]. FBG was inscribed into a MM PMMA POF using He-Cd laser at $325 \mathrm{~nm}$ and into a SM GOF using Argon laser at $244 \mathrm{~nm}$. The PMMA POF had a core diameter of $10 \mu \mathrm{m}$ and a cladding thickness of $130 \mu \mathrm{m}$. At a constant temperature of $25^{\circ} \mathrm{C}$, the POFs showed a linear response to the relative humidity in the range of $50-95 \%$ with a sensitivity of $35.2 \mathrm{pm} \cdot \%^{-1}$, 
while for the GOFs, a sensitivity of only $0.28 \mathrm{pm} \cdot \%^{-1}$ was achieved. At constant humidity, the POFs still performed better than GOFs in measuring temperature with a sensitivity of $-55 \mathrm{pm} \cdot{ }^{\circ} \mathrm{C}^{-1}$ compared to $13.9 \mathrm{pm} \cdot{ }^{\circ} \mathrm{C}^{-1}$ for the GOFs. Hysteresis is the major problem using FBG PMMA POFs in the monitoring of relative humidity-up to 30 minutes response time was required to reach the full response. This can be partially resolved by etching a fiber's cladding to speed up the diffusion of the water into the core [73].

The drawback for polymer FBG sensors is also obvious, the commonly used PMMA POF can only absorb moisture up to $0.3 \mathrm{wt} \%$ [74]. When using polymer FBG sensors to monitor temperature, strain or stress in the air, the measurements need to be calibrated against the relative humidity at same time. This cross-sensitivity imposes a significant drawback to the implementation of polymer FBG sensors in the field $[75,76]$. Recently, a new type of polymer material-cyclic olefin copolymers $\left(\right.$ TOPAS $^{\oplus}$ ) - has drawn considerable attention from researchers due to its low affinity towards water and chemical inert nature [74]. High quality Bragg gratings were first successfully inscribed into TOPAS ${ }^{\circledR} \mathrm{mPOF}$ by Johnson et al. [77]. By using a He-Cd laser, FBG was incorporated into a SM TOPAS mPOF with a Bragg wavelength of $1567.7 \mathrm{~nm}$. When the TOPAS ${ }^{\circledR} \mathrm{mPOF}$ based FBG sensor was examined at an environment with a constant relative humidity of $55 \%$, the sensor showed a linear response to the temperature variation from 20 to $35^{\circ} \mathrm{C}$ and a sensitivity of $-36.5 \mathrm{pm} \cdot{ }^{\circ} \mathrm{C}^{-1}$. However, the authors also pointed out that one of the limitations of using TOPAS $^{\circ}$ is its low glass transition temperature $\left(78^{\circ} \mathrm{C}\right), 30^{\circ} \mathrm{C}$ less than PMMA. Yuan et al. [78], from the same research group, examined the influence of humidity on TOPAS ${ }^{\oplus} \mathrm{mPOF}$ based FBG sensor in measuring strain and temperature. In addition to having the operating wavelength set at the conventional Bragg wavelength of $1568 \mathrm{~nm}$, the authors also fabricated a FBG that operated between $800-900 \mathrm{~nm}$ to reduce the power loss in the fiber. At $870 \mathrm{~nm}$, the sensitivity of the TOPAS ${ }^{\varpi}$ mPOF FBG sensor was determined to be $0.64 \mathrm{pm} \cdot \mu \mathrm{p}^{-1}$ (up to $2.17 \%$ strain) and -78 $\mathrm{pm} \cdot{ }^{\circ} \mathrm{C}^{-1}\left(23^{\circ} \mathrm{C}\right.$ to $\left.32.6^{\circ} \mathrm{C}\right)$. The strain sensitivity is similar to the PMMA based FBG sensor reported previously $[64,65]$, while the sensitivity in temperature was doubled [77], which was due to the smaller diameter of the fiber used. The humidity tests illustrated that the TOPAS ${ }^{\circledast}$ FBG sensor responded linearly to the humidity change. For the TOPAS FBG sensor operated at $849 \mathrm{~nm}$, sensitivity was observed to peak at 0.7 $\mathrm{pm} \cdot \%^{-1}$ when decreased the relative humidity from $90 \%$ to $50 \%$. For the TOPAS ${ }^{\oplus}$ FBG sensor operated at the Bragg wavelength of $1568 \mathrm{~nm}$, maximum of sensitivity of $-0.59 \mathrm{pm} \cdot \%^{-1}$ was observed when the relative humidity was increased from $30 \%$ to $90 \%$.

A polymer FBG strain sensor can be easily converted into an accelerometer. Stefani et al. developed an accelerometer using a SM PMMA mPOF based FBG sensor [79]. FBG was incorporated into a mPOF with Bragg wavelengths of $1550 \mathrm{~nm}$ and $850 \mathrm{~nm}$ using a phase mask technique. Then, an accelerometer prototype was prepared by using a fork-shaped mechanical transducer to convert the acceleration into strain through elongation of the fiber caused by acceleration. The accelerometer showed a frequency response up to $1 \mathrm{kHz}$ and the resonance frequency was found to be $3 \mathrm{kHz}$. At a resonance wavelength of $1550 \mathrm{~nm}$, a linear sensitivity of $19 \mathrm{pm} \cdot \mathrm{g}^{-1}$ up to $15 \mathrm{~g}$ of acceleration was achieved; while for the $850 \mathrm{~nm} \mathrm{mPOF}$, the sensitivity was found to be $7.6 \mathrm{pm} \cdot \mathrm{g}^{-1}$. Comparing the $\mathrm{mPOF}$ and GOF FBG accelerometers fabricated by the same method showed that the MPOF sensor achieved more than four times greater sensitivity over its counterpart at both Bragg wavelengths.

\section{Microstructured POF sensors}

Microstructured optical fibers (MOFs), also as known as photonic crystal fibers (PCFs), are a newly emerging type of waveguide that can guide the light through hollow cores running longitudinally along the fiber. The MOFs can be divided into several categories based on their geometries and light guiding mechanisms, including photonicband gap fiber, holey fiber, hole-assisted fiber and Bragg fiber [80,81]. Employing MOFs to develop optic sensors have several prominent advantages over conventional solid core fibers. Indicators or sensing layers adopted in the hollow cores inside the MOFs provide significantly more sensing areas and stronger interactions with the transmitted light waves than the conventional fibers. The location of sensing elements is isolated from the surrounding environment and hence minimizes any undesirable external interference. mPOFs offer additional benefits over their glass counterparts due to their ease and versatility of fabrication techniques and intrinsic tailorability of the polymers $[82,83]$.

$\mathrm{Li}$ and Wang et al. reported a mPOF sensor using Rhodamine 6G (Rh 6G) as the indicator for the detection of hydrogen peroxide and nitrites $[84,85]$. Li and Wang first used a titanium oxide film as the carrier for Rh $6 \mathrm{G}$ to detect the hydrogen peroxide [84]. The acidic solution containing tetrabutyl titanate, ethanol and Rh 6G was drawn into the hollow holes to form a three-dimensional network of titanium dioxide with entrapped $\mathrm{Rh} 6 \mathrm{G}$. It was discovered that the resulting sensor responded to hydrogen peroxide in an acidic solution containing certain concentrations of potassium iodide. A linear response over a range of $1.6 \times 10^{-7} \mathrm{~mol} \cdot \mathrm{L}^{-1}$ to $9.6 \times 10^{-5} \mathrm{~mol} \cdot \mathrm{L}^{-1}$ was recorded and the interference caused by the inorganic ions was very limited. Later, $\mathrm{Li}$ and Wang used cellulose acetate as the carrier for Rh $6 \mathrm{G}$ to detect the nitrites through a similar fabrication technique [85].

mPOFs were also used to fabricate gas sensors with a considerable increase in sensitivities compared to conventional FOSs. Wang et al. reported a carbon dioxide gas sensor composed of 547 hollow channels which were coated with porous ethyl cellulose layers doped with phenol red [86]. In order to achieve reversible measurements, cetyltrimethyl ammonium hydroxide $(\mathrm{CTAOH})$ was added into the film as the phase-transfer agent. The resulting sensing film contains an ion pair of quaternary ammonium and deprotonated phenol red, which allows for a reversible reaction between phenol red and carbon dioxide [87]. The sensor showed a linear response towards carbon dioxide mixed in nitrogen over a range of $0-20 \mathrm{v} / \mathrm{v} \%$ and the detection limit was determined to be $0.5 \mathrm{v} / \mathrm{v} \%$. The sensor reproducibility was found to be stable over 8 cycles of repeated measurements and only acidic gases were found to produce major interference. Later, Yang employed a sol-gel technique for the fabrication of a mPOF oxygen sensor with ruthenium (II) chloride as the indicator [88]. The ruthenium compound was coated on the exterior surface of a conventional PMMA fiber for the detection of oxygen [49]. The fabricated sensor exhibited a linear response to oxygen from $0-100 \mathrm{v} / \mathrm{v} \%$ with a $50 \mathrm{~ms}$ response time. By replacing the ruthenium compound with eosin, Peng et al. developed an ammonia gas sensor using a similar fabrication technique [89]. The sensors' response range can be tailored through a hexadecyl trimethyl ammonium bromide (CTAB) co-entrapment process and the limit of detection was found to be as low as $50 \mathrm{ppm}$.

\section{Localized surface plasmon resonance based sensors}

The sensitivity of intrinsic-transmission fiber optic sensors is largely dependent on the intensity of the evanescent wave (EW). Even though the EW intensity can be enhanced by varying the geometry of the fibers, the interaction between the EW and trace analyte amounts is frequently 
too weak to obtain reliable sensing results. To resolve this problem, a novel detection scheme-localized surface plasmon resonance (LSPR) has been adopted to improve the intrinsic-transmission systems, especially in the field of biological analyte sensing.

A full description of the LSPR effect has been explained in literature [90-92], hence only a compact version of the relevant theories will be discussed herein. Prior to elucidating the theories of LSPR, it is important to distinguish LSPR from surface plasmon resonance (SPR). SPR and LSPR effects can both be attributed to the photo-driven coherent oscillations of free electrons on the surface of a metal substrate surrounded by a dielectric medium. The fundamental difference between SPR and LSPR is the location of the electrons. SPR happens on a thin metal film while LSPR occurs on the surface of a nanoparticle. Compared to SPR, LSPR is highly sensitive to the local dielectric environment variations within a much shorter range from the metal surface (40-50 times less than SPR), which makes it an ideal candidate for the detection of ultra-low concentrations of bio-analytes $[93,94]$.

Theoretical explanations for the LSPR effect were provided by Gustav Mie who derived analytical solutions from the Maxwell's equation [95]. According to Mie, a perfect spherical nanoparticle was assumed to be surrounded by a homogeneous medium which can be described by bulk optical dielectric functions, hence the light extinction properties $\left(\sigma_{\text {ext }}\right)$ of the nanoparticles can be attributed to absorption $\left(\sigma_{\text {abs }}\right)$ and scattering $\left(\sigma_{\text {sca }}\right)$. If the particle size is significantly smaller than the wavelength of the incident light $(\mathrm{r} / \lambda<0.1)$, then absorption dominates the equation (Equation. 4) [44].

$$
\sigma_{\mathrm{ext}}=\frac{24 \pi^{2} \mathrm{Nr}^{3} \varepsilon_{\mathrm{m}}^{3 / 2}}{\lambda \ln (10)}\left[\frac{\varepsilon_{\mathrm{i}}(\lambda)}{\left(\varepsilon_{\mathrm{r}}(\lambda)+\chi \varepsilon_{\mathrm{m}}\right)^{2}+\varepsilon_{\mathrm{i}}(\lambda)^{2}}\right]
$$

Where, $\varepsilon_{\mathrm{m}}$ is the dielectric constant of the surrounding medium, $\varepsilon_{\mathrm{r}}$ and $\varepsilon_{\mathrm{i}}$ represent the real and imaginary components of the particles' dielectric functions, respectively. $\mathrm{N}$ is the electron density of the particle and $r$ is the particle radius. $\chi$ reflects the shape of the particle, it equals 2 in the case of a sphere and larger values will be assigned if the particle has higher aspect ratios [96]. Here, $\varepsilon_{\mathrm{r}}$ can be further expressed in the following equation [44]:

$$
\varepsilon_{\mathrm{r}}=1-\frac{\omega_{\mathrm{p}}^{2}}{\omega^{2}+\gamma^{2}}
$$

Where, $\omega_{\mathrm{p}}$ and $\gamma$ present the plasmon frequency and damping parameter of the particle, respectively. If $\varepsilon_{\mathrm{r}}$ is close to $-2 \varepsilon_{\mathrm{m}}$ (the resonance conditions), the dominator in Equation 4 would be minimized and the electromagnetic (EM) field is enhanced compared to the incident field. Equation 5 can be simplified if the resonance frequency occurs in the visible and near-infrared regions $\left(\omega_{p}>>\gamma\right)$ [44]:

$$
\varepsilon_{\mathrm{r}}=1-\frac{\omega_{\mathrm{p}}^{2}}{\omega^{2}}
$$

By substituting $\varepsilon_{\mathrm{r}}=-2 \varepsilon_{\mathrm{m}}, \lambda=\frac{2 \pi \mathrm{c}}{\omega}$ and $\varepsilon_{\mathrm{m}}=\mathrm{n}_{\mathrm{m}}^{2}$ into Equation 6 , we can obtain [44]:

$$
\lambda_{\text {max }}=\lambda_{\mathrm{p}} \sqrt{2 \mathrm{n}_{\mathrm{m}}^{2}+1}
$$

Where, $\lambda_{\max }$ is the maximum absorption frequency of the LSPR band immersed in the medium which has the refractive index of $\mathrm{n}_{\mathrm{m}}$, and $\lambda_{p}$ corresponds to the plasmon wavelength of the particle. According to Equation 7, the relationship between a LSPR absorption peak and the refractive index of the surrounding medium can be approximated to be linear over a small range of indices. The sensitivity (S) of a LSPR sensor based on the shift of the plasmon peaks $(\mathrm{nm})$ with respect to the change of the medium refractive index can be defined as:

$$
\mathrm{S}=\frac{\delta \lambda}{\delta \mathrm{n}_{\mathrm{m}}}\left[\mathrm{nm} \cdot \mathrm{RIU}^{-1}\right]
$$

For a LSPR POF biosensor, the sensitivity can also be defined as:

$$
\mathrm{S}=\frac{\delta \lambda}{\delta \mathrm{c}}\left[\mathrm{nm} \cdot \mathrm{L} \cdot \mathrm{mol}^{-1}\right]
$$

Where, $\mathrm{c}$ stands for the concentration of the biological analytes.

Under the aforementioned resonance conditions, Equation 4 can be further simplified to become:

$$
\sigma_{\text {ext }}=\frac{24 \pi^{2} \mathrm{Nr}^{3} \varepsilon_{\mathrm{m}}^{3 / 2}}{\lambda \ln (10) \varepsilon_{\mathrm{i}}(\lambda)}
$$

It is clear that the extinction coefficient of the particle also responds to variations in the refractive index of the surrounding medium due to the term $\left(\varepsilon_{\mathrm{m}}^{3 / 2}\right)$ in the Equation 10.

Both LSPR peak shift and absorption variations with respect to the medium's refractive index have been employed for the fabrication of LSPR POF sensors. However, due to the intrinsically higher accuracy of the wavelength determination by modern instrumentation and the minimal interference caused by extraneous optical power fluctuations, LSPR peak shift based detection mechanisms have become more prevalent in recent advancements of LSPR optical sensors [44,93,97].

Compared to the booming applications of LSPR GOF sensors in recent years, the development of LSPR POF sensors is severely hindered, which mainly resulted from the difficulty of employing sol-gel techniques onto POF surface. Among the handful of LSPR POF sensors reported, the majority of them are based on the same configurations of LSPR GOF sensors with little improvements.

Chen et al. developed a LSPR POF sensor to detect the concentration of hydrofluoric acid (HF) [98]. A thin layer of silicon dioxide bearing amine moieties was first attached onto the surface of a PMMA POF using a typical sol-gel technique. Then AuNPs were immobilized onto the silicon layer via electrostatic adsorption. The attached AuNPs layer was subsequently covered with a layer of silicon oxide matrix that originated from tetramethoxysilane (TMOS) as the sensitive coating. The HF detection mechanism is based on the intensity variations of the output light signals when the sensitive coating on AuNPs is etched by HF. Although, the author's initial incentive of using PMMA POF was to avoid the decomposition of fibers by HF that would occur for the GOFs, the use of a silicon layer for the attachment of AuNPs still resulted in the destruction of the sensor after prolong exposure.

Recently, a LSPR POF sensor was reported by Cennamo et al. for the detection of TNT using molecularly imprinted polymers (MIP) as the sensing layer [99]. Five-branched gold nanostars (AuNSs) were first dispersed in a pre-polymeric mixture that contained TNT as the template molecule. Then, the mixture was dropped onto a side polished PMMA POF where the polymerization was carried out at elevated temperatures. The template molecules were subsequently extracted by washing with ethanol, leaving specific binding sites for TNT. AuNSs have much better sensitivity than AuNPs due to their high aspect ratios, this translated into high sensitivity for the reported sensor. The sensor sensitivity was determined to be $8.3 \times 10^{5} \mathrm{~nm} \cdot \mathrm{mol}^{-1}$ for a tapered POF, which is thirty times more sensitive compared to the TNT sensors reported previously [99]. 
Jin et al. incorporated thiol and amine functionalities onto the surface of PMMA POFs through direct surface chemical modifications, allowing for subsequent covalent bonding or electrostatic adsorption of colloidal AuNPs [100]. It was discovered that the sensitivity of the LSPR POF sensor was substantially affected by the chemistries employed for the AuNP immobilization. AuNPs immobilized on both thiolated PMMA POFs showed higher sensitivities compared to aminated PMMA POFs. Afterwards, Jin et al. functionalized surface immobilized AuNPs with glycopolymer brushes carrying glucose moieties for the detection of concanavalin A (Con A) [101]. The "glycocluster effect" induced by pendent carbohydrate moieties enabled a stronger affinity for Con A binding, which resulted in a dramatic expansion of the sensors' response range. It was reported that, the limit of detection of the LSPR sensor was $1.3 \mathrm{nmol} \cdot \mathrm{L}^{-1}$ with a saturated response at 1054.2 nmol. $\mathrm{L}^{-1}$

Similar to LSPR POF sensors, POFs have been successfully employed in the fabrication of surface plasmon resonance (SPR) optical sensors. The conventional SPR optical sensors are based on Otto configuration [102] and later improved Kretschmann configuration [103]. However, these prism based configurations suffer complex mechanical and optical structure, bulky size and difficult to miniaturize $[104,105]$. These problems have driven researcher to use optical fibers as the host for SPR since both prism and optical fiber can generate EW at surface due to TIF. Some experimental and theoretical work have been reported recent years on SPR sensors (mostly based on GOFs), showing a great potential of this new generation fiber optic SPR sensors [104-108]

Cennamo et al. adapted classic fabrication technique of SPR GOF sensors in development SPR POF sensors [109]. In the first configuration, a layer of gold film, $40 \mathrm{~nm}$ thick, was sputtered coated onto cladding stripped PMMA POF. In the second configuration, microposit S1813 photoresist was dip coated onto the fiber before gold sputter coating. The experimental test results showed, with the photoresist buffer layer, the proposed sensor has a greater linear response range from 1.332 to 1.418. While, the response range for the sensor without buffer layer was considerably lower due to light dissipation. The resolution and signal to noise ratio (SNR) were found also higher for the sensor with the buffer layer. In the refractive index medium of 1.354, resolution was found to be 0.00059 RIU and sensitivity was determined to be 2533 $\mathrm{nm} \cdot \mathrm{RIU}^{-1}$. With the successful fabrication of POF based SPR sensor, Cennamo later demonstrated the great potential of this sensor in the field of detecting biomolecules and aqueous chemicals [110]. By immobilisation of transglutaminase (tTG) on the gold layer, the SPR POF sensor was able to detect antitransglutaminase (anti-tTG) in the range of $30 \mathrm{nM}$ and $3000 \mathrm{nM}$. On the other hand, by coating a layer of molecular imprinted polymer (MIP) on the gold layer, the proposed SPR POF sensor has the capable of specific monitoring of trinitrotoluene (TNT) and L-nicotine $[111,112]$.

Besides using SI polymer fibers, SPR also has been incorporated into PCF or mPOF. Theoretical evaluation the performance of SPR PCF sensor was reported by $\mathrm{Lu}$ et al. [113]. By using finite element method (FEM), a large-mode-area PCF with a thin layer of silver coated outside shows the size and number of air holes have limited effect on the sensitivity, while both spectral and intensity resolution were found in the range of $8.3 \times 10^{-5}$ and $9.4 \times 10^{-5}$ RIU. About the same time, Wang et al. reported a SPR sensor based on a side-hole mPOF [114122]. Unlike previous techniques that deposited metals from the fluid onto the inner surface of holes, they sputter coated a gold layer onto the core of slotted fiber. Two SPR frequencies were observed at $560 \mathrm{~nm}$ and
$620 \mathrm{~nm}$ when the sensor was tested in the fluids of refractive indices of 1.38 and 1.41, respectively. The sensitivity was estimated to be 2000 $\mathrm{nm} \cdot \mathrm{RIU}^{-1}[120-122]$.

\section{Conclusion}

This paper has presented a brief review of the recent progress of POF sensors. The main thrust of employing POFs originated from their high flexibility, high tensile strain, good gas and liquid permeability and in particular, versatile surface functionalization pathways. A wide range of sensing technologies has been successfully implemented in the fabrication of POFs FOSs, such as monolayer graphene, FBG, LSPR/SPR and acoustic/ultrasound. However, it is also noted that some POF sensors fabrication methods (e.g. sol-gel techniques) were directly transferred from GOF sensors without utilizing POFs' unique properties, which resulted in similar or worsen sensing performance. Therefore, further work is still required to fully explore POFs potential in the field of FOSs.

\section{Acknowledgement}

The authors would like to acknowledge the University of New South Wales (UNSW), Faculty of Engineering Early Career Researcher Faculty Research Grant (ECR/FRG) for funding of this research.

\section{References}

1. Hunt J (2008) A new way to monitor using fibreoptics. Sens Rev 28: 199-204.

2. Zubia J, Arrue J (2001) Plastic Optical Fibers: An Introduction to Their Technological Processes and Applications. Opt Fiber Technol 7: 101-140.

3. Perrone G, Vallan A (2008) A Displacement Measurement System Based on Polymer Optical Fibres. Meas Sci Technol 17: 5.

4. Steiger U (1998) Sensor properties and applications of POF. Paper presented at the 7th Conference on Sensors and Their Applications. Dublin, Ireland.

5. Zubia J, Aresti O, Arrúe J, Lopez-Amo M (1999) Barrier sensor based on plastic optical fiber to determine the wind speed at a wind generator in 8th International Conference on Plastic Optical Fibres and Applications-POF, China, Japan.

6. Zhou Q, Tabacco MB, Rosenblum KW (1991) Development of Chemical Sensors Using Plastic Optical Fiber. Paper presented at the SPIE Plastic Optical Fibers Boston, MA.

7. Toba E (1999) Fiber Optic Fluorosensor for Oxygen measurement. Paper presented at the 16th Instrumentation and Measurement. Technology Conference, IEEE, Venice, Italy.

8. Bilro L, Alberto N, Pinto JL, Nogueira R (2012) Optical sensors based on plastic fibers. Sensors (Basel) 12: 12184-12207.

9. Kurihara K, Ohkawa H, Iwasaki Y, Niwa O, Tobita T, et al. (2004) Fiber-optic conical microsensors for surface plasmon resonance using chemically etched single-mode fiber. Anal Chim Acta 523: 165-170.

10. Kalymnios D, Scully P, Zubia J, Poisel H (2002) POF Sensors. Paper presented at the POF Reprint Series, Boston, MA.

11. Udd E, Spillman WB (2011) Fiber Optic Sensors: An Introduction for Engineers and Scientists. Wiley.

12. López-Higuera JM (2002) Handbook of Optical Fibre Sensing Technology. Wiley

13. Grattan K, Sun T (2000) Fiber optic sensor technology: an overview. Sens Actuators A 82: 40-61.

14. Bartlett RJ, Philip-Chandy R, Eldridge P, Merchant DF, Morgan R, et al. (2000) Plastic optical fibre sensors and devices. Trans Inst Meas Control 22: 431-457.

15. Leung A, Shankar PM, Mutharasan R (2007) A review of fiber-optic biosensors Sens Actuators B 125: 688-703.

16. Peters K (2011) Polymer optical fiber sensors-a review. Smart Mater Struct 20: 013002.

17. Méndez A, Morse TF (2007) Specialty Optical Fibres Handbook. Elsevier Inc Burlington, USA. 
Citation: Jin Y, Granville AM (2016) Polymer Fiber Optic Sensors - A Mini Review of their Synthesis and Applications. J Biosens Bioelectron 7: 194. doi:10.4172/2155-6210.1000194

Page 9 of 11

18. Kuzyk MG (2007) Polymer Fiber Optics: Materials, Physics, and Applications. Taylor and Francis Press, Boca Raton.

19. Garvey DW, Zimmerman K, Young P, Tostenrude J, Townsend JS, et al. (1996) Single-mode Nonlinear-Optical Polymer Fibers. J Opt Soc Am B 13: 2017-2023.

20. Kaino T, Jinguji K, Nara S (1982) low loss Poly (methyl methacrylate-d5) Core fibres. Appl Phys Lett 41: 802-804.

21. Klein LC (1988) Sol-gel technology for thin films, fibers, preforms, electronics, and speciality shapes. Noyes publications.

22. Mac Craith B, Mc Donagh C, McEvoy A, Butler T, O'keeffe G, et al. (1997) Optical chemical sensors based on sol-gel materials: Recent advances and critical issues. J Sol-Gel Sci Technol 8: 1053-1061.

23. Fabbri P, Pilati F, Rovati L, McKenzie R, Mijovic J (2011) Poly (ethylene oxide)-silica hybrids entrapping sensitive dyes for biomedical optical $\mathrm{pH}$ sensors: Molecular dynamics and optical response. Opt Mater 33: 1362-1369.

24. Schyrr B, Pasche S, Scolan E, Ischer R, Ferrario D, et al. (2013) Development of a polymer optical fiber $\mathrm{pH}$ sensor for on-body monitoring application. Sens Actuators B.

25. Kulkarni A, Lee JH, Nam JD, Kim T (2010) Thin film-coated plastic optical fiber probe for aerosol chemical sensing applications. Sens Actuators, B 150: 154-159.

26. Chang CP, Chao CY, Huang JH, Li AK, Hsu CS, et al. (2004) Fluorescent conjugated polymer films as TNT chemosensors. Synth Met 144: 297-301.

27. Chu F, Yang J (2012) Coil-shaped plastic optical fiber sensor heads for fluorescence quenching based TNT sensing. Sens Actuators A 175: 43-46.

28. Novoselov KS, Fal'ko VI, Colombo L, Gellert PR, Schwab MG, et al. (2012) A roadmap for graphene. Nature 490: 192-200.

29. Rao CN, Sood AK, Subrahmanyam KS, Govindaraj A (2009) Graphene: the new two-dimensional nanomaterial. Angew Chem Int Ed Engl 48: 7752-7777.

30. Loh KP, Bao Q, Ang PK, Yang J (2010) The chemistry of graphene. J Mater Chem 20: 2277-2289.

31. Jiang DE, Cooper VR, Dai S (2009) Porous graphene as the ultimate membrane for gas separation. Nano Lett 9: 4019-4024.

32. Bunch JS, Verbridge SS, Alden JS, van der Zande AM, Parpia JM, et al. (2008) Impermeable atomic membranes from graphene sheets. Nano Lett 8: 2458-2462.

33. Novoselov KS, Geim AK, Morozov SV, Jiang D, Zhang Y, et al. (2004) Electric field effect in atomically thin carbon films. Science 306: 666-669.

34. Lee C, Wei X, Kysar JW, Hone J (2008) Measurement of the elastic properties and intrinsic strength of monolayer graphene. Science 321: 385-388.

35. Kim JA, Hwang T, Dugasani SR, Amin R, Kulkarni A, et al. (2013) Graphene based Fiber Optic Surface Plasmon Resonance for Bio-chemical Sensor Applications. Sens Actuators B 187: 426-433.

36. Kulkarni A, Kim H, Amin R, Park SH, Hong BH, et al. (2012) A novel method for large area graphene transfer on the polymer optical fiber. $\mathrm{J}$ Nanosci Nanotechnol 12: 3918-3921.

37. Zhang H, Kulkarni A, Kim H, Woo D, Kim YJ, et al. (2011) Detection of acetone vapor using graphene on polymer optical fiber. J Nanosci Nanotechnol 11: 5939-5943.

38. Some S, Xu Y, Kim Y, Yoon Y, Qin H, et al. (2013) Highly sensitive and selective gas sensor using hydrophilic and hydrophobic graphenes. Sci Rep 3: 1868.

39. O'Neill HJ, Gordon SM, O'Neill MH, Gibbons RD, Szidon JP (1988) A computerized classification technique for screening for the presence of breath biomarkers in lung cancer. Clin Chem 34: 1613-1618.

40. Kharitonov SA, Barnes PJ (2002) Biomarkers of some pulmonary diseases in exhaled breath. Biomarkers 7: 1-32.

41. Peng G, Tisch U, Adams O, Hakim M, Shehada N, et al. (2009) Diagnosing lung cancer in exhaled breath using gold nanoparticles. Nat Nanotechnol 4 : 669-673

42. Nath N, Anand S (1998) Evanescent wave fiber optic fluorosensor: effect of tapering configuration on the signal acquisition. Opt Eng 37: 220.

43. Kuswandi B, Andres R, Narayanaswamy R (2001) Optical fibre biosensors based on immobilised enzymes. Analyst 126: 1469-1491.

44. Mayer KM, Hafner JH (2011) Localized surface plasmon resonance sensors. Chem Rev 111: 3828-3857.

45. Lopatynskyi AM, Lopatynska OG, Jay Guo L, Chegel VI (2011) Localized surface plasmon resonance biosensor-Part I: Theoretical study of sensitivityextended Mie approach. IEEE Sens J 11: 361-369.

46. Rahman H, Harun S, Yasin M, Phang S, Damanhuri S, et al. (2011) Tapered plastic multimode fiber sensor for salinity detection. Sens Actuators A 171: 219-222.

47. Poisson A (1980) The concentration of the $\mathrm{KCl}$ solution whose conductivity is that of standard seawater (35\%)at $15^{\circ} \mathrm{C}$. IEEE J Oceanic Eng 5: 24-28.

48. Pulido C, Esteban O (2013) Tapered polymer optical fiber oxygen sensor based on fluorescence-quenching of an embedded fluorophore. Sens Actuators B 184: 64-69.

49. Chu F, Yang J, Cai H, Qu R, Fang Z (2009) Characterization of a dissolved oxygen sensor made of plastic optical fiber coated with ruthenium-incorporated solgel. Appl Opt 48: 338-342.

50. Carraway E, Demas J, DeGraff B, Bacon J (1991) Photophysics and photochemistry of oxygen sensors based on luminescent transition-metal complexes. Anal Chem 63: 337-342.

51. Da Silva AV, de Souza NCC, Miguel MAL, Beres C, Yugue ES, et al. (2008) Development of a biosensor based in polymericoptical fiber to detect cells in water and fluids. Paper presented at the 19th International Conference on Optical Fibre Sensors.

52. Wandermur G, Rodrigues D, Allil R, Queiroz V, Peixoto R, et al. (2014) Plastic optical fiber-based biosensor platform for rapid cell detection. Biosens Bioelectron 54: 661-666.

53. Chapman $P(2000)$ Methods available for the detection of Escherichia coli O157 in clinical, food and environmental samples. World J Microbiol Biotechnol 16: 733-740.

54. Kashyap R (1999) Fiber Bragg Gratings. Academic Press, Burlington, MA

55. Hill KO, Meltz G (1997) Fiber Bragg grating technology fundamentals and overview. J Lightwave Technol 15: 1263-1276.

56. Chiavaioli F, Trono C, Giannetti A, Brenci M, Baldini F (2014) Characterisation of a label-free biosensor based on long period grating. J Biophotonics 7: 312-322.

57. Chiavaioli F, Biswas P, Trono C, Bandyopadhyay S, Giannetti A, et al. (2014) Towards sensitive label-free immunosensing by means of turn-around point long period fiber gratings. Biosensors Bioelectron 60: 305-310.

58. Chiavaioli F, Trono C, Giannetti A, Brenci M, Baldini F (2014) Label-Free Biosensor Based on Copolymer-Functionalized Optical Fiber Long-Period Grating in Sensors and Microsystems Pp: 199-203.

59. Xiong Z, Peng G, Wu B, Chu P (1999) Highly tunable Bragg gratings in singlemode polymer optical fibers. IEEE Photonics Technol Lett 11: 352-354.

60. Liu H, Peng G, Chu P (2002) Polymer fiber Bragg gratings with 28-dB transmission rejection. IEEE Photonics Technol Lett 14: 935-937.

61. Rajan G, Noor MY, Lovell NH, Ambikaizrajah E, Farrell G, et al. (2013) Polymer micro-fiber Bragg grating. Opt Lett 38: 3359-3362.

62. Kalli K, Dobb HL, Webb DJ, Carroll K, Komodromos M, et al. (2007) Electrically tunable Bragg gratings in single-mode polymer optical fiber. Opt Lett 32: 214-216.

63. Tao X, Yu J, Tam H (2007) Photosensitive polymer optical fibres and gratings Trans Inst Meas Control 29: 255-270.

64. Stefani A, Yuan W, Markos C, Bang O (2011) Narrow bandwidth 850-nm fiber Bragg gratings in few-mode polymer optical fibers. IEEE Photonics Technol Lett 23: 660-662.

65. Johnson IP, Kalli K, Webb DJ (2010) 827 nm Bragg grating sensor in multimode microstructured polymer optical fibre. Electron Lett 46: 1217-1218.

66. Sáez-Rodríguez D, Nielsen K, Rasmussen HK, Bang O, Webb DJ (2013) Highly photosensitive polymethyl methacrylate microstructured polymer optical fiber with doped core. Opt Lett 38: 3769-3772.

67. Statkiewicz-Barabach G, Tarnowski K, Kowal D, Mergo P, Urbanczyk W (2013) Fabrication of multiple Bragg gratings in microstructured polymer fibers using a phase mask with several diffraction orders. Opt Express 21: 8521-8534. 
Citation: Jin Y, Granville AM (2016) Polymer Fiber Optic Sensors - A Mini Review of their Synthesis and Applications. J Biosens Bioelectron 7: 194. doi:10.4172/2155-6210.1000194

68. Luo Y, Yan B, Li M, Zhang X, Wu W, et al. (2011) Analysis of multimode POF gratings in stress and strain sensing applications. Opt Fiber Technol 17: 201-209.

69. Luo Y, Wu W, Wang T, Cheng X, Zhang Q, et al. (2012) Analysis of multimode BDK doped POF gratings for temperature sensing. Opt Commun 21: 4353-4358.

70. Gu F, Yu H, Fang W, Tong L (2013) Nanoimprinted polymer micro/nanofiber Bragg gratings for high-sensitive strain sensing. IEEE Photonics Technol Lett 25: 22-24.

71. Chen X, Zhang C, Webb DJ, Peng GD, Kalli K (2010) Bragg grating in a polymer optical fibre for strain, bend and temperature sensing. Meas Sci Technol 21 : 094005.

72. Zhang C, Zhang W, Webb DJ, Peng GD (2010) Optical fibre temperature and humidity sensor. Electron Lett 46: 643-644.

73. Zhang W, Webb DJ, Peng GD (2012) Investigation into time response of polymer fiber Bragg grating based humidity sensors. J Lightwave Technol 30 : 1090-1096.

74. Khanarian G, Celanese H (2001) Optical properties of cyclic olefin copolymers. Opt Eng 40: 1024-1029.

75. Harbach GN, Limberger HG, Salathé RP (2010) Influence of Humidity and Temperature on Polymer Optical Fiber Bragg Gratings. Paper presented at the Advanced Photonics \& Renewable Energy, Karlsruhe.

76. Zhang ZF, Tao XM (2012) Synergetic effects of humidity and temperature on PMMA based fiber Bragg gratings. J Lightwave Technol 30: 841-845.

77. Johnson IP, Yuan W, Stefani A, Nielsen K, Rasmussen HK, et al. (2011) Optical fibre Bragg grating recorded in TOPAS cyclic olefin copolymer. Electron Lett $47:$ 271-272.

78. Yuan W, Khan L, Webb DJ, Kalli K, Rasmussen HK, et al. (2011) Humidity insensitive TOPAS polymer fiber Bragg grating sensor. Opt Express 19: 1973119739.

79. Stefani A, Andresen S, Yuan W, Herholdt-Rasmussen N, Bang O (2012) High sensitivity polymer optical fiber-Bragg-grating-based accelerometer. IEEE Photonics Technol Lett 24: 763-765.

80. Knight JC (2003) Photonic crystal fibres. Nature 424: 847-851.

81. Russell PSJ (2006) Photonic-crystal fibers. J Lightwave Technol 24: 4729-4749.

82. Eijkelenborg MA, Argyros A, Barton G, Bassett IM, Fellew M, et al. (2003) Recent progress in microstructured polymer optical fibre fabrication and characterisation. Opt Fiber Technol 9: 199-209.

83. Large MC, Blacket D, Bunge CA (2010) Microstructured polymer optical fibers compared to conventional POF: Novel properties and applications. IEEE Sens J10: 1213-1217.

84. Li D, Wang L (2010) Fluorescence hydrogen peroxide probe based on a microstructured polymer optical fiber modified with a titanium dioxide film. Appl Spectrosc 64: 514-519.

85. Li D, Wang L (2010) Cellulose acetate polymer film modified microstructured polymer optical fiber towards a nitrite optical probe. Opt Commun 283: 2841 2844.

86. Wang J, Wang L (2010) Carbon dioxide gas sensor derived from a 547-hole microstructured polymer optical fiber preform. Opt Lett 35: 3270-3272.

87. Mills A, Lepre A, Wild L (1997) Breath-by-breath measurement of carbon dioxide using a plastic film optical sensor. Sens Actuators B 39: 419-425.

88. Yang X, Peng L, Yuan L, Teng P, Tian F, et al. (2011) Oxygen gas optrode based on microstructured polymer optical fiber segment. Opt Commun 284: 3462-3466.

89. Peng L, Yang X, Yuan L, Wang L, Zhao E, et al. (2011) Gaseous ammonia fluorescence probe based on cellulose acetate modified microstructured optical fiber. Opt Commun 284: 4810-4814.

90. Maier SA (2007) Plasmonics: fundamentals and applications: fundamentals and applications. Springer Science \& Business Media, Bath, UK.

91. Schasfoort RB, Tudos AJ (2008) Handbook of Surface Plasmon Resonance. Royal Society of Chemistry, Cambridge, UK.

92. Hutter E, Fendler JH (2004) Exploitation of localized surface plasmon resonance. Adv Mater 16: 1685-1706.
93. Willets KA Van Duyne RP (2007) Localized surface plasmon resonance spectroscopy and sensing. Annu Rev Phys Chem 58: 267-297.

94. Whitney AV, Elam JW, Zou S, Zinovev AV, Stair PC, et al. (2005) Localized surface plasmon resonance nanosensor: a high-resolution distancedependence study using atomic layer deposition. J Phys Chem B 109: 20522 20528.

95. Mie G (1908) Beitrage zur Optik trüber Medien, speziell kolloidaler Metallösungen. Annalen Der Physik 330: 377-445.

96. Link S, El-Sayed MA (1999) Spectral properties and relaxation dynamics of surface plasmon electronic oscillations in gold and silver nanodots and nanorods. J Phys Chem B 103: 8410-8426.

97. Petryayeva E, Krull UJ (2011) Localized surface plasmon resonance: nanostructures, bioassays and biosensing-a review. Anal Chim Acta 706: 8-24.

98. Chen IC, Lin SS, Lin TJ, Du JK (2011) Detection of hydrofluoric acid by a SiO sol-gel coating fiber-optic probe based on reflection-based localized surface plasmon resonance. Sensors (Basel) 11: 1907-1923.

99. Cennamo N, Donà A, Pallavicini P, D'Agostino G, Dacarro G, et al. (2015) Sensitive detection of, 4, 6-trinitrotoluene by tridimensional monitoring of molecularly imprinted polymer with optical fiber and five-branched gold nanostars. Sens Actuators B 208: 291-298.

100. Jin Y, Wong KH, Granville AM (2016) Developing Localized Surface Plasmon Resonance Biosensor Chips and Fiber Optics via Direct Surface Modification of PMMA Optical Waveguides. Colloids Surf Physicochem Eng Aspects.

101. Jin Y, Wong KH, Granville AM (2016) Enhancement of Localized Surface Plasmon Resonance polymer based biosensor chips using well-defined glycopolymers for lectin detection. J Colloid Interface Sci 462: 19-28.

102. Otto A (1968) Excitation of nonradiative surface plasma waves in silver by the method of frustrated total reflection. Z Phys 216: 398-410.

103. Kretschmann E, Raether H (1968) Radiative decay of non radiative surface plasmons excited by light (Surface plasma waves excitation by light and decay into photons applied to nonradiative modes). Zeitschrift Fuer Naturforschung Teil A 23: 2135

104.Gupta B, Verma R (2009) Surface plasmon resonance-based fiber optic sensors: principle, probe designs, and some applications. J Sens 2009.

105. Sharma AK, Jha R, Gupta B (2007) Fiber-optic sensors based on surface plasmon resonance: a comprehensive review. IEEE Sens J7: 1118-1129.

106. Homola J (2008) Surface plasmon resonance sensors for detection of chemical and biological species. Chem Rev 108: 462-493.

107. Abdulhalim I, Zourob M, Lakhtakia A (2008) Surface plasmon resonance for biosensing: a mini-review. Electromagnetics 28: 214-242.

108. Hassani A, Skorobogatiy M (2007) Design criteria for microstructured-opticalfiber-based surface-plasmon-resonance sensors. J Opt Soc Am B 24: 1423-1429.

109. Cennamo N, Massarotti D, Conte L, Zeni L (2011) Low cost sensors based on SPR in a plastic optical fiber for biosensor implementation. Sensors (Basel) 11: $11752-11760$

110. Cennamo N, Varriale A, Pennacchio A, Staiano M, Massarotti D, et al. (2012) An innovative plastic optical fiber-based biosensor for new bio/applications. The Case of Celiac Disease, Sens Actuators B.

111. Cennamo N, Agostino G, Galatus R, Bibbò L, Pesavento M, et al. (2013) Sensors based on surface plasmon resonance in a plastic optical fiber for the detection of trinitrotoluene. Sens Actuators B 188: 221-226.

112. Cennamo N, D’Agostino G, Pesavento M, Zeni L (2014) High selectivity and sensitivity sensor based on MIP and SPR in tapered plastic optical fibers for the detection of I-nicotine. Sens Actuators B 191: 529-536.

113. Lu Y, Hao CJ, Wu BQ, Musideke M, Duan LC, et al. (2013) Surface plasmon resonance sensor based on polymer photonic crystal fibers with metal nanolayers. Sensors (Basel) 13: 956-965.

114. Wang A, Docherty A, Kuhlmey BT, Cox FM, Large MC (2009) Side-hole fiber sensor based on surface plasmon resonance. Opt Lett 34: 3890-3892.

115. McDonagh C, Maccraith BD, McEvoy AK (1998) Tailoring of sol-gel films for optical sensing of oxygen in gas and aqueous phase. Anal Chem 70: 45-50.

116. Guo L, Ni Q, Li J, Zhang L, Lin X, et al. (2008) A novel sensor based on 
Citation: Jin Y, Granville AM (2016) Polymer Fiber Optic Sensors - A Mini Review of their Synthesis and Applications. J Biosens Bioelectron 7: 194. doi:10.4172/2155-6210.1000194

the porous plastic probe for determination of dissolved oxygen in seawater. Talanta 74: 1032-1037.

117. Zhou Q, Shahriari MR, Kritz D, Sigel Jr GH (1988) Porous fiber-optic sensor for high-sensitivity humidity measurements. Anal Chem 60: 2317-2320.

118. Lamela H, Gallego D, Gutierrez R, Oraevsky A (2011) Interferometric fiber optic sensors for biomedical applications of optoacoustic imaging. J Biophotonics 4: 184-192.

119. Wild G, Hinckley S (2008) Acousto-ultrasonic optical fiber sensors: overview and state-of-the-art. IEEE Sens J8: 1184-1193.
120. Culshaw B, Thursby G, Betz D, Sorazu B (2008) The detection of ultrasound using fiber-optic sensors. IEEE Sens J8: 1360-1367.

121. Gallego D, Lamela $H(2009)$ High-sensitivity ultrasound interferometric singlemode polymer optical fiber sensors for biomedical applications. Opt Lett 34 1807-1809.

122. Gallego D, Lamela H (2011) High sensitivity interferometric polymer optical fiber ultrasound sensors for optoacoustic imaging and biomedical application. Paper presented at the 21st International Conference on Optical Fiber Sensors, Ottawa, Canada. 